\title{
The TBOSS (Taurus Boundary of Stellar/Substellar) Survey of Disk Properties
}

\author{
J. Patience ${ }^{1,2}$, J. Bulger ${ }^{2}$, K. Ward-Duong ${ }^{1}$, H. Bouy ${ }^{3}$, C. Pinte ${ }^{4}$, \\ F. Ménard ${ }^{4}$, J.-L. Monin ${ }^{5}$, J. Koda ${ }^{6}$ and C. D. Dowell ${ }^{7}$ \\ ${ }^{1}$ Arizona State University, ${ }^{2}$ The University of Exeter, ${ }^{3}$ Centro de Astrobiología, ${ }^{4}$ Universidad \\ de Chile, ${ }^{5}$ Institut de Planétologie et d Astrophysique (IPAG), ${ }^{6}$ Stony Brook University, \\ ${ }^{7}$ California Institute of Technology
}

\begin{abstract}
With the combination of Herschel PACS (far-IR), submillimeter (submm) and millimeter $(\mathrm{mm})$ ground-based observations, we are leading an investigation of all members with spectral types M4 and onwards in the Taurus star-forming region. This complete census spans the stellar to substellar boundary (M6.25), and the wavelength range covers the transition from optically thick to optically thin emission. From our Hershel PACS observations of $\sim 135$ sources, we obtain a detection rate of $\sim 40 \%$ at $70 \mu \mathrm{m}$ and a detection rate of $\sim 20 \%$ at $160 \mu \mathrm{m}$, and provide the first far-IR measurements for the majority of these members. With our complementary submm and $\mathrm{mm}$ observations, best fit SED models from the radiative transfer code MCFOST will be used to infer disk properties such as scale height, mass, outer radius and maximum dust grain size. These comprehensive population statistics of disks are critical for testing star/brown dwarf and planet formation models around these later type members of Taurus.
\end{abstract}

Keywords. stars: low-mass, brown dwarfs

\section{Background}

Taurus represents an important population of young stars and brown dwarfs for which detailed investigations of disk frequencies and properties are possible. The presence of disks around stellar and substellar objects has been used to argue for a common formation mechanism or alternatively differences in the dynamical history compared to higher mass stars (Scholz \& Jayawardhana 2008; Thies \& Kroupa 2007).

\section{Sample \& Observations}

There are 151 known members in Taurus that have spectral types of M4 or later, corresponding to low mass stars and brown dwarfs (Luhman et al. 2010). A complete census of Hershel PACS observations at $70 \mu \mathrm{m}$ and $160 \mu \mathrm{m}$ has been obtained for these members. The members comprise of 67 Class I and II objects and 71 Class III objects. From extensive observations with single dish ground based observations in the submillimeter with the Caltech Submillimeter Obervatory (CSO), and in the millimeter with IRAM, we also have obtained observations at $350 / 450 \mu \mathrm{m}$, and/or $1.2 \mathrm{~mm}$ for 30 low mass stars and brown dwarf members. High-resolution observations for 24 of the 151 Taurus members detected in the Herschel program will be observed at $850 \mu \mathrm{m}$ with ALMA in Cycle 1.

\section{Initial Results \& Future Work}

The number of Herschel PACS detections of this survey are shown in Fig. 1. Far-IR and submm maps are shown for CIDA-1, along with a modelled SED fit, obtained using the 

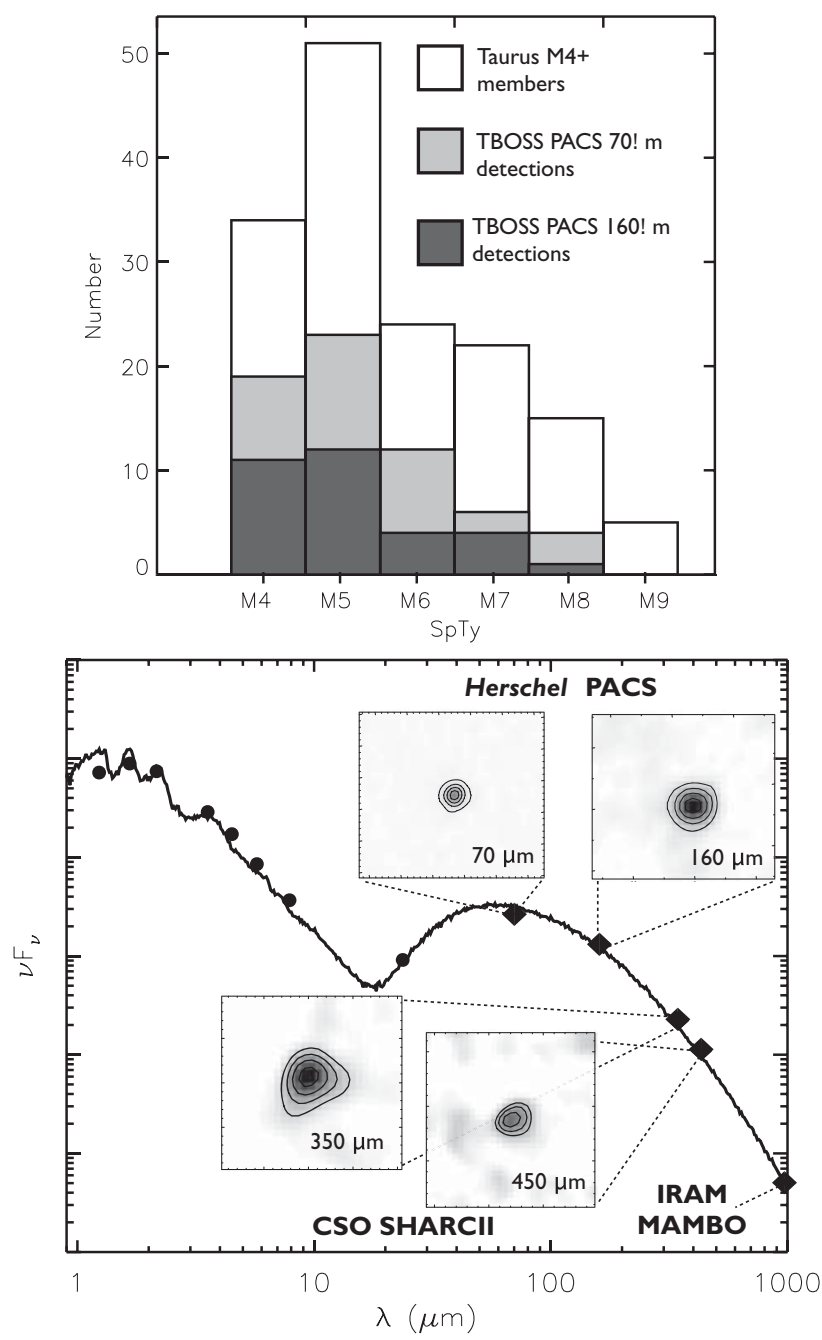

Figure 1. (top:) Histogram of all Taurus members with spectral types M4 and greater, showing the number of Herschel PACS detections at $70 \mu \mathrm{m}$ and $160 \mu \mathrm{m}$. (bottom:) SED model fit for CIDA-1 (M5.5) obtained using the radiative transfer code MCFOST. Observations have been obtained in the far-IR with Herschel PACS at $70 \mu \mathrm{m}$ and $160 \mu \mathrm{m}$, in the submm with the CSO at $350 \mu \mathrm{m}$ and $450 \mu \mathrm{m}$ and in the millimeter with IRAM at $1.2 \mathrm{~mm}$ for this target. Well sampled, multi-wavelength observations across the far-IR to millimeter wavelengths are essential in the breaking degeneracies amongst disk parameters (e.g. the redundancy of disk mass and outer radius).

radiative transfer code MCFOST (Pinte et al. 2006). Our multi-wavelength observations will provide a benchmark, detailed study of the structure of disks encircling low mass stars and brown dwarfs.

\section{References}

Luhman, K. L., Allen, P. R., Espaillat, C., Hartmann, L., \& Calvet, N. 2010, ApJS, 186, 11

Pinte, C., Ménard, F., Duchêne, G., \& Bastien, P. 2006, A $\& A$, 459, 797

Scholz, A. \& Jayawardhana, R. 2008, ApJ, 672, L49

Thies, I. \& Kroupa, P. 2007, ApJ, 671, 767 